CHEMICAL BIOLOGY

\section{Bring them back alive}

\author{
Michael Yarus
}

\section{A deep search has turned up an RNA that can carry out the chemically complex 'aldol' reaction involved in sugar metabolism. Could this be similar to an ancestral catalyst that existed billions of years ago?}

The title of this piece evokes the capture of exotic beasts for zoos, and hints at the reanimation of the extinct. Both goals apply to the work of Fusz et al. ${ }^{1}$, who, as they report in Chemistry and Biology, went hunting for a sort of molecule that might have existed about 4 billion years ago. According to the 'RNA world' hypothesis, life at this time consisted of macro-molecular or cellular assemblies, with RNA molecules rather than proteins catalysing chemical reactions. In support of this, a number of RNA enzymes (ribozymes) have been created, or captured from the wild, that can carry out the sorts of chemical reaction - the formation of peptide bonds, for example - that would have been necessary for such life.

Now Fusz et al. identify a ribozyme that can catalyse the particularly tricky aldol reaction. This involves carbon-carbon bond formation which would have been required at several significant points in the evolution of life, including, possibly, when ribose was first produced. Fusz and colleagues' success at finding a ribozyme that could catalyse the aldol reaction (Fig. 1) makes it more likely that the 'organisms' in the RNA world actually used aldol reactions ${ }^{2}$.

The authors used selection-amplification, or SELEX ${ }^{3}$, to track down their prey. This technique sifts through a quadrillion or so random sequences of RNA to find a few (or perhaps just one) that can carry out a specified reaction. This may seem like looking for a needle in a haystack, but SELEX is extremely powerful: it has turned up more RNA catalysts than have been found by harvesting from living catalytic diversity. effective evolutionary search. material ${ }^{4}$. Aldol chemistry occurs in a crucial step in sugar metabolism in all cells, and is central to glycolysis, the Calvin cycle, and other energy-yielding pathways. As only the second reaction forming a carbon-carbon bond found to be carried out by $\mathrm{RNA}^{5,6}$, aldol reactions are also a welcome addition to RNA

In nature, novel activities would probably arise from the adaptation of existing molecules that already had some of the required properties. By contrast, SELEX usually involves a single search of a set of randomized sequences. The number of sequences expands exponentially with the size of the molecule found - ten times for each 1.6 nucleotides added ${ }^{7}$. It is much easier for a novel activity to evolve in nature, through the selection of subtle enhancements of an existing molecule, than for us to happen upon an entirely new activity all at once by finding a sequence of $15-20$ nucleotides. Thus, when single-step selection succeeds, it strongly implies that the active site could also have been found by ribocytes, the supposed primordial RNA 'cells', using a more

Fusz et al. ensured that the desired aldol catalyst would announce itself by coaxing the aldol reaction to link a tag to the active RNA. The tag then aided the physical separation of the RNA from the substrate so that the RNA could be studied in isolation. The result of their search was a ribozyme that could carry out an aldol reaction about 4,300-fold faster than the reaction would occur in solution. This catalyst was difficult to find: there was only one sequence that could carry out the
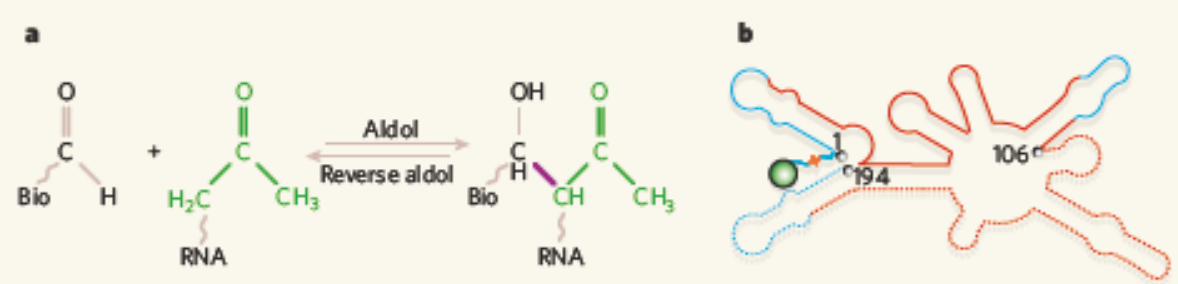

Figure 1 | Resurrecting an aldol riboryme. a, Fusz et al. ${ }^{1}$ tested a vast array of random-sequence RNAs to see whether any of them could speed up this predicted variant of the aldol reaction. The new carbon-carbon bond produced is shown in purple, and the reactive 'ketone' substrate group is shown in green. Bio is biotin, a chemical tag that allows the molecule to be easily separated from the reaction mixture. b, The selected ribozyme. The numbers represent nucleotides counting from the 5 ' end. Blue shows fixed sequences and red shows the sequences that were randomized. Broken lines show those nucleotides that can be deleted without destroying the molecule's activity. The green sphere is the ketone substrate, and the orange zigzag shows the light-sensitive link, used to ensure that biotin addition has occurred at the ketone, and not elsewhere. reaction efficiently among the $10^{15}$ starting RNAs. This unique large RNA had a major nucleotide tract that could be deleted without affecting the aldol reaction (Fig. 1). This is to be expected given the single-pass nature of the experiment - had an aldol ribozyme evolved naturally, this 'junk' would probably have been whittled away by the selection.

The new ribozyme absolutely requires zinc ions, which are also uniquely sufficient for the RNA's activity (magnesium, barium, copper, manganese and many other metals will not, on their own, activate ribozymes). This is notable because, of the two common types of protein enzyme that carry out aldol reactions in modern cells, one uses zinc as a catalytic element. So the RNA found by Fusz et al. may resemble an existing protein in the way it polarizes its reactants to speed up the aldol reaction.

Finding an aldol ribozyme is provocative because an RNA world would have required some form of energy metabolism; energy must be captured to maintain complex and unstable molecules such as RNA. Rudimentary catalysts for the replication ${ }^{8}$ of RNA or the synthesis of proteins ${ }^{9}$ were isolated some time ago. But very few RNAs, either natural or recently winnowed out by artificial selection, supply clues to how energy might have been harvested in an RNA world. So there is a void even in purely imaginative accounts of the RNA world, which these findings help to fill.

The reverse aldol (retroaldol) reaction (Fig. 1) is interesting in this light because although not itself an energy-harvesting reaction, it enhances the energy yield from hexose sugars such as glucose during glycolysis. In today's biology, it is closely coupled to reactions that yield ATP, the energy currency for most cellular work. The new reaction is thus a potentially useful part, if not the heart, of a possible metabolic RNA engine that would eat carbohydrates.

Michael Yarus is in the Department of Molecular, Cellular and Developmental Biology, University of Colorado, Boulder, Colorado 80309-0347, USA. e-mail:yarus@colorado.edu

1. Fusz, S, Eisenfulur, A, Srivatsan, S. G, Heckel, A. \& Famulok M.Chem. Bial.12,941-950 (2005).

2. Helm, M , Petermelec, M, Ge, B, Fiammenga, R\& läschike, A.J. Am Chem. Soc. 127,10492-10493(2005).

3 Wilson, D.S.\& Szostak, I. W. Amu Rex Biochem.68, 611-647 (1999)

4. Lilley, D.M. I. Curr. Opin Struct. Bial. 15, 313-323(2005).

5. Yanus, M. \& Knight, R in The Genetic Codeand the Origin of Lfe(ed. de Pouplana, L) 75-91 (Landes Bioscience, Georgetown, Texas, 2004)

6. Yanus, M. Annu. Rex Blochem. 74, 179-198 (2005).

7. Tarasow, T.M. Tassow, S.L. \& Eaton, B. E Nature 389, 54-57 (1997).

8. Lawrence, M.S.\& Bartel, D.P. RNA n,1173-1180 (2005)

9. Yanus, M. Cald Sping Harb. Symp Quant. Bial.66,207-215 (2001).

\section{Correction}

In "Ecology: Roots of stability" by Peter D. Moore (Nature 437, 959-961; 2005), the reference to the main paper discussed was incorrect. The correct reference is Kahmen, A., Perner, J. \& Buchmann, N. Funct. Ecol. 19,594-601(2005). 